\title{
A NEW AUTOMATIC SYSTEM CALIBRATION OF MULTI-CAMERAS AND LIDAR SENSORS
}

\author{
M. Hassanein a, *, A. Moussa ${ }^{\text {a,b }}$, N. El-Sheimy ${ }^{\text {a }}$ \\ ${ }^{a}$ Department of Geomatics Engineering, University of Calgary, Calgary, Alberta, Canada - (mohamed.hassanein2, amelsaye, \\ elsheimy)@ucalgary.ca \\ ${ }^{\mathrm{b}}$ Department of Electrical Engineering, Port-Said University, Port-Said, Egypt
}

Commission I, ICWG I/Va

KEY WORDS: System Calibration, LIDAR, Multi-Camera, Point Cloud

\begin{abstract}
:
In the last few years, multi-cameras and LIDAR systems draw the attention of the mapping community. They have been deployed on different mobile mapping platforms. The different uses of these platforms, especially the UAVs, offered new applications and developments which require fast and accurate results. The successful calibration of such systems is a key factor to achieve accurate results and for the successful processing of the system measurements especially with the different types of measurements provided by the LIDAR and the cameras. The system calibration aims to estimate the geometric relationships between the different system components. A number of applications require the systems be ready for operation in a short time especially for disasters monitoring applications. Also, many of the present system calibration techniques are constrained with the need of special arrangements in labs for the calibration procedures. In this paper, a new technique for calibration of integrated LIDAR and multi-cameras systems is presented. The new proposed technique offers a calibration solution that overcomes the need for special labs for standard calibration procedures. In the proposed technique, 3D reconstruction of automatically detected and matched image points is used to generate a sparse imagesdriven point cloud then, a registration between the LIDAR generated 3D point cloud and the images-driven 3D point takes place to estimate the geometric relationships between the cameras and the LIDAR.. In the presented technique a simple 3D artificial target is used to simplify the lab requirements for the calibration procedure. The used target is composed of three intersected plates. The choice of such target geometry was to ensure enough conditions for the convergence of registration between the constructed 3D point clouds from the two systems. The achieved results of the proposed approach prove its ability to provide an adequate and fully automated calibration without sophisticated calibration arrangement requirements. The proposed technique introduces high potential for system calibration for many applications especially those with critical logistic and time constraints such as in disaster monitoring applications.
\end{abstract}

\section{INTRODUCTION}

Recently, a wide range of mapping systems integrating different sensors have been developed. Examples of such integrated systems have been deployed on Unmanned Arial Vehicles (UAVs) in the last few years. The different uses of the UAVs offered a wide range of new applications and developments which require fast and accurate results (Lin et al., 2011; Vallet et al., 2011). One of the important sensors combination which takes huge attention is the integration of multi-cameras and the LIDAR as these sensors provide complementary information when fusing their data (Douillard et al., 2007). The successful calibration of such integrated systems is a key factor to achieve accurate results and for achieving the successful processing of the system measurements. System calibration aims to estimate the geometric relationships among the different system components. A number of applications require the systems be ready for operation in a short time especially those used for disasters monitoring applications. Also, many of the present system calibration techniques are constrained with the need of special arrangements in labs for the calibration procedures.

Away from the techniques that require special laboratory arrangements for system calibration, there are several other techniques to estimate calibration parameters between the LIDAR and cameras. These techniques can be classified into two main categories (Kwak et al., 2011). The first category uses a direct search for the LIDAR beam within the image. However, these techniques require IR cameras to detect the LIDAR spots on the images directly (Kwak et al., 2010). The other category indirectly estimates the correspondences between common features observed from the two sensors. This approach can be performed in 3D as proposed by (Zhang and Pless, 2004). In this approach the main idea is to use a known pose of a calibration target in 3D and the calibration parameters can be estimated by solving an optimization problem to minimize the position error of the LIDAR data with respect to the calibration target in 3D (Kwak et al., 2011).

Different indirect techniques have been proposed depending on simple targets, see for example (Kwak et al., 2011). However, these techniques require the collection of high number of images from different ranges which might not be always easy to achieve specially for time constrained applications. Also, with the same concept of indirect approaches, (Rodriguez et al., 2008) used an artificial target and determined the relative position of the sensors by finding the features correspondence and solving a classical target based registration.

This paper introduces a new technique for calibrating a system composed of multi-cameras and a LIDAR. While the described system in this paper is composed of a LIDAR and two cameras, the proposed technique can be easily, and with more effective

\footnotetext{
* Corresponding author
} 
results, extended to multi-cameras systems that has more than two cameras. The system used in this paper, integrate a LIDAR and two cameras. It is assumed that the multi-cameras system is already calibrated and the output is referenced to one of the cameras which is the master camera. The proposed technique estimates the geometric relation between the LIDAR unit and the master camera.

There are different techniques that can be used to perform the system calibration for systems composed of LIDAR and cameras. The most straight forward system calibration techniques depend on having well equipped laboratory and use manual measurements for the calibration. The proposed system calibration technique provides a simple alternative as it provides the ability to perform system calibration with very simple procedures. The proposed technique depends on using a simple target where no need to know the exact geometry of the used target. Another advantage of the proposed technique is its ability to perform the calibration by using just two images which can be easily acquired in a shorter time compared to other calibration techniques.

It is important to note that the proposed technique has two main advantages: (a) the simplicity of the used target which enables a more convenient calibration, and (b) Avoiding the need for precise point to point correspondence, as the registration step can be effectively employed without such point to point correspondence. This proposed calibration approach depends on matching the two 3D point clouds created from the LIDAR and cameras instead of matching specific points which might need more effort.

The outline of the paper is as follows: In section 2 we describe two related topics to our work which are the construction of 3D point cloud and the Iterative Closest Point (ICP). Section 3 introduces the proposed methodology of the proposed calibration technique. Section 5 discusses the results. Finally the conclusions are presented in Section 6.

\section{RELATED WORK}

In this section an introduction to two related topics in the proposed technique is provided. First, the construction of the 3D point cloud is introduced followed by a brief introduction to the Iterative Closest Point (ICP).

\subsection{D Point Cloud Construction}

The proposed technique for the multi-cameras and LIDAR system calibration depends on having two 3D point clouds, generated from the LIDAR and the images, for a specific target. The 3D point cloud is the representation of the measured points by the sensor in a $3 \mathrm{D}$ coordinates system referenced to the sensor frame. For the LIDAR system, it is straight forward process to generate 3D point cloud as the LIDAR sensor main output is the $3 \mathrm{D}$ coordinates for the measured objects. Regarding the multicameras, generating the 3D point cloud from the cameras output, require the calculation of the $3 \mathrm{D}$ coordinates of the measured objects using the space intersection technique.

This intersection step requires detecting matched points in the different images and depending on the intrinsic parameters of the cameras, acquired form the camera calibration, and the extrinsic parameters of the two cameras, acquired from the multi-cameras system calibration, the $3 \mathrm{D}$ coordinates of the matched points from the cameras images can be calculated and so the 3D point cloud for the multi-cameras system can be generated.

\subsection{Iterative Closest Point (ICP)}

The main idea of the proposed technique for the system calibration is to estimate the transformation parameters between the two 3D point clouds generated from the two used sensors as discussed in the previous section. Iterative Closest Point (ICP) is a registration technique proposed by (Besl \& McKay, 1992), where exact point-to-point correspondence between 3D point clouds is not required (George Vosselman, 2010). If we have two 3D point clouds $\left(x_{i}\right),\left(y_{i}\right)$, the rigid body transformation can be described as the following equation.

$$
y_{i}=R x_{i}+y_{0}
$$

Where:

$(R)$ is the rotation between the two point clouds

$\left(y_{0}\right)$ is the translation between the two point clouds

The main objective of the ICP registration is to rigidly transform the slave point cloud $\left(x_{i}\right)$ to best fit the master point cloud $\left(y_{i}\right)$ such that the sum of square Euclidean distance $\left(e^{2}\right)$ between closest points is minimized (Vosselman \& Maas, 2010).

$$
e^{2}=\sum_{i}\left\|R x_{i}+y_{0}-y_{i}\right\|^{2}
$$

The summation in this registration is taken over all the data points. The main limitation of this registration technique is the need for a good initial alignment of the two point clouds. The roughly estimated manual measurements of the calibration parameters are used to initialize the registration step of the proposed approach.

\section{SYSTEM OVERVIEW}

In this section, the description of the proposed technique for the system calibration is presented. Figure (1) shows a flowchart for the proposed system calibration main steps. The first step of the approach is to generate the images-driven 3D point cloud. This step starts with extracting and matching feature points between the two image using Speeded Up Robust Features (SURF) technique, the matching is refined using the Random Sample Consensus (RANSAC) algorithm. With the help of the intrinsic and extrinsic orientation parameters of the cameras, the matched feature points are intersected to build a sparse point cloud. Another point cloud of the target is acquired using the laser scanner. The final step of the proposed system calibration technique is the ICP registration to register the two $3 \mathrm{D}$ point clouds obtained in the previous step. As mentioned before, the main goal of this step is to estimate the transformation parameters between laser scanner coordinate system and the master camera coordinate system. So, the estimated transformation/registration parameters represent the calibration parameters for the system.

The estimated calibration parameters include the lever-arm transition vector and the boresight rotation matrix, which represent the geometric relations between the two main systems of the used sensor system. The target field used in the proposed technique consists of three planes target with different normal vector for each plane to provide enough constraints during the ICP registration step. It is important to note that the three planes of the target need to be rich with visual features to be detected in order to create the $3 \mathrm{D}$ point cloud from the camera images.

Also, it must be pointed that with a sensor as the Velodyne laser scanner, the volume of the target has an important effect. As with a very small target when it is positioned away from the laser scanner, it is possible to miss the whole target so, it is 
recommended to use suitable volume of the target that matches the distance to the laser scanner.

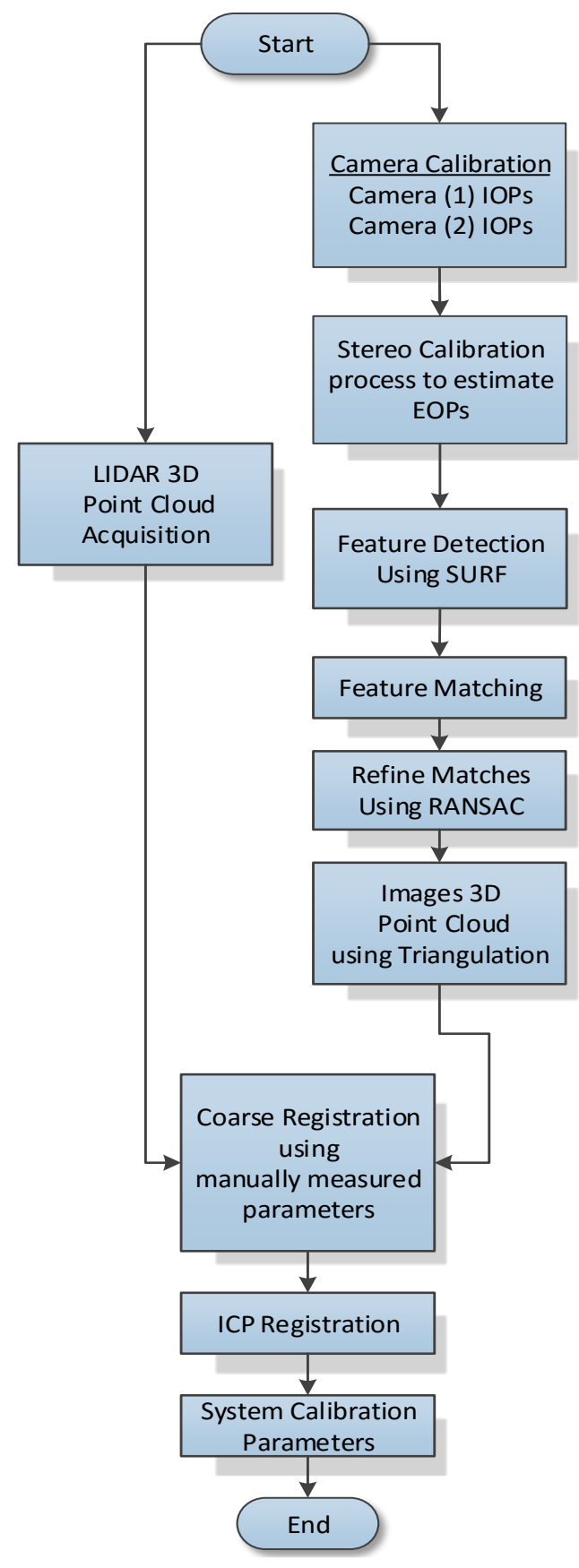

Figure 1. System overview

\section{EXPERMINTALS RESULTS}

In the following sections, a full description of the testing procedure of the proposed technique is introduced.

\subsection{Sensors Description}

To test the proposed calibration technique, a system integrating the following sensors is used (a) the Velodyne HDL-32E LIDAR is used to provide the 3D LIDAR point cloud, and (b) two Canon PowerShot S110 cameras. The sensors were tested in two different scenarios; (a) the LIDAR system was almost in the middle distance between the two cameras, while the two cameras where rotated towards each other as shown in figure (2), and (b) the LIDAR system was also almost in the middle between the two cameras, while the two cameras were almost horizontally parallel to each other as shown in figure (3). The used target is a simple cardboard box which three planes of its six surfaces were covered with coloured features as shown in figure (4).

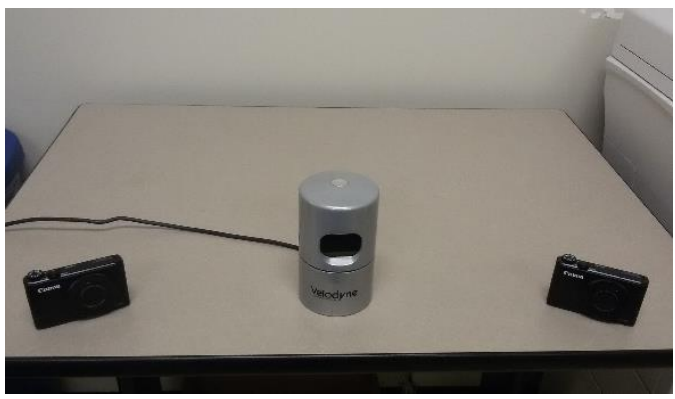

Figure 2. The first scenario for the sensors

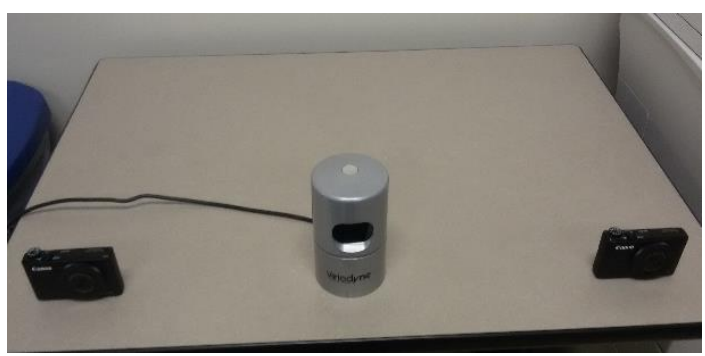

Figure 3. The second scenario for the sensors

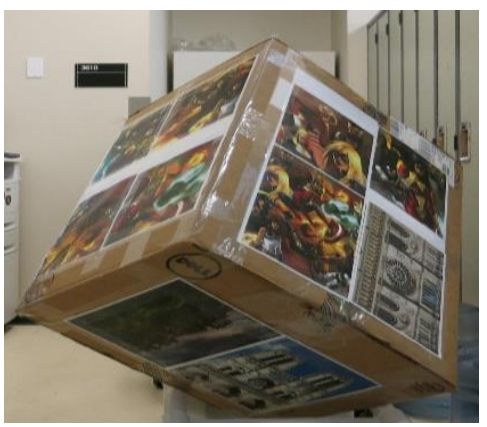

Figure 4 . The used target for the testing

\subsection{Collecting Data}

To test the proposed method, four data sets were collected from the described system for the used targets. Two data sets were collected for each scenario. For each data set, LIDAR data were collected for the target to create the 3D point cloud from the laser scanner. Also, two images for the same target from the two used cameras were used to create the $3 \mathrm{D}$ point cloud from the cameras.

In the second scenario, the used target was placed in two different distances from the sensors to test the effect of the distance between the used target and the sensors on the calibration accuracy.

\subsection{D Point Clouds}

The collected data sets were used to construct the two 3D point clouds needed for the calibration of each scenario. Four 3D LIDAR point clouds were acquired using the Velodyne HDL- 
32E laser scanner. Figure (5) shows a sample LIDAR point cloud of the target.

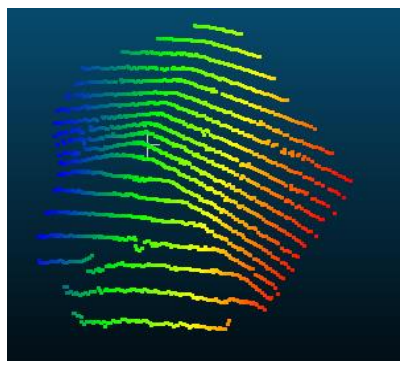

Figure 5. LIDAR 3D point cloud of the target
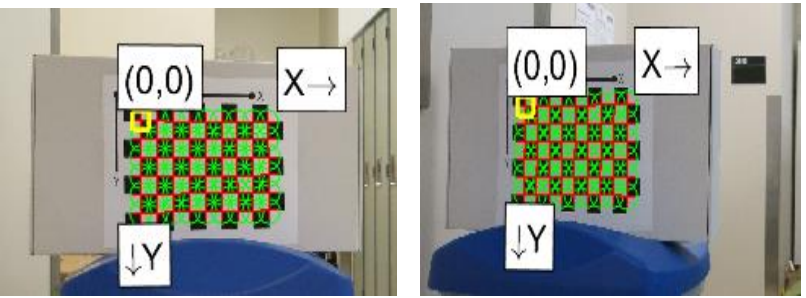

Figure 6 . The checker board technique for camera calibration

For constructing the $3 \mathrm{D}$ point cloud from the multi-cameras sensor, the images were used as following. First, for each scenario, a camera calibration procedure was performed to estimate the intrinsic parameters of each camera. The calibration was done using the checker board technique (Heikkila and Silvén, 1997; Zhang, 2000) which depends on using a checker board with known dimensions and use a detection procedure for the intersection points within the board as shown in figure (6). Using detected points and the known distances of the checker board, a separate camera calibration procedure was performed to estimate the intrinsic parameters of each camera.

For calibrating each camera the used checker board was positioned in different 8 positions with different orientation as shown in figure (7). For each position of the checker board an image was captured from each camera for the checker board. The same procedures were followed for the second scenario of the system, but with dealing with only 6 positions for the checker board.

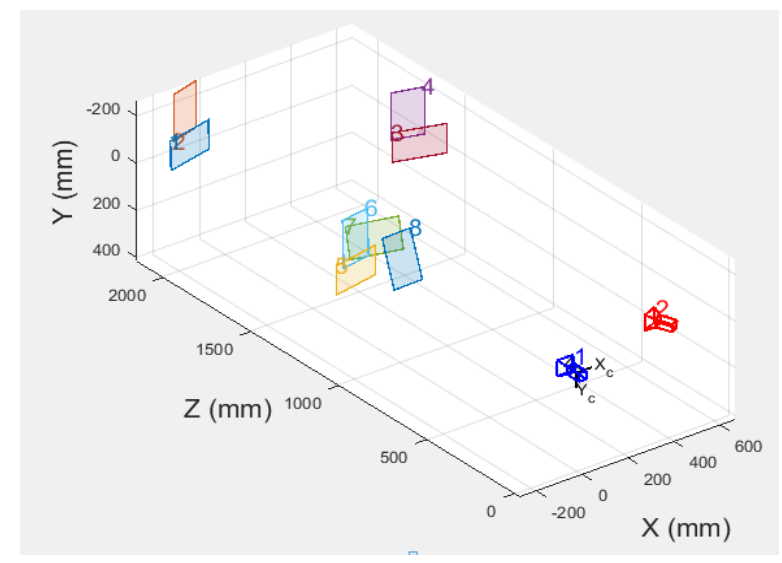

Figure 7. Extrinsic cameras visualization

Then, a system calibration for the multi-cameras system was conducted to estimate the extrinsic parameters of the multi- cameras system. The multi-cameras system calibration was conducted using the same images of the checker board used for each camera calibration. Finally, for each data set two images for the target are captured from the two cameras.

For constructing the images-driven 3D point cloud, it is important to make sure that the three surfaces appear in the captured images of the target. SURF feature detection technique (Bay et al., 2008) has been used to detect the interest features on the three surfaces of the target and match them in the two images to construct the $3 \mathrm{D}$ point cloud from the two images for the used target as shown in figure (8).

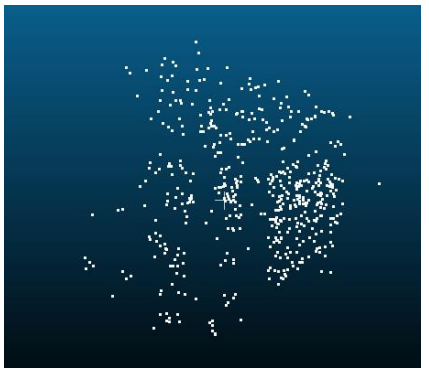

Figure 8 . The images-driven 3D point cloud of the target

\subsection{Estimating the Calibration Parameters}

The testing procedures were conducted on two main stages. The first stage was performed to get the system calibration parameters for each scenario. This step was done by using the constructed 3D point clouds and using the ICP to register the two point clouds. The registration parameters represent the system calibration parameters which are the lever-arm translation vector and the rotation boresight matrix.

The constructed 3D point clouds from the laser scanner and the images for the first and the third data sets were used in the ICP registration to estimate the system calibration parameters for the first and the second scenarios. The second stage of testing the proposed technique was to use the estimated system calibration parameters to register the 3D point cloud acquired from the laser scanner. The mean and the standard deviation of the differences between the registered 3D LIDAR point cloud and the 3D images point cloud, were calculated. The calculated mean and standard deviation were used as an indication of the error in the calibration parameters. This step was done for the two scenarios using the second and fourth data sets and the results are shown in table (1).

\begin{tabular}{|c|c|c|c|c|}
\hline \multirow{2}{*}{ Scenario } & \multicolumn{2}{|c|}{ Scenario (1) } & \multicolumn{2}{c|}{ Scenario (2) } \\
\cline { 2 - 5 } & $\begin{array}{c}\text { Mean } \\
(\mathrm{m})\end{array}$ & $\operatorname{Std}(\mathrm{m})$ & $\begin{array}{c}\text { Mean } \\
(\mathrm{m})\end{array}$ & Std (m) \\
\hline $\begin{array}{c}\text { Before } \\
\text { calibration }\end{array}$ & 0.1067 & 0.061985 & 0.2960 & 0.1314 \\
\hline $\begin{array}{c}\text { After } \\
\text { calibration }\end{array}$ & 0.066 & 0.06484 & 0.0644 & 0.0514 \\
\hline
\end{tabular}

Table 1. The mean and the standard deviation of the distance between the LIDAR and images-driven point clouds before the calibration and after the calibration 


\section{RESULTS}

The two systems scenarios were used in the testing, each system scenario was used to collect two data sets. The first data set was used to estimate the system calibration parameters while the second data set was used to test the estimated calibration parameters. The results from table (1), figure (9) for the first scenario, and figure (10) for the second scenario show the ability of the proposed technique to adequately estimate the calibration parameters. It is important to note that the used data were collected using minimum sensors configuration and with the minimum number of images needed to construct $3 \mathrm{D}$ point cloud from the multi-cameras systems which is two images.

As the proposed technique proved its ability to provide adequate system calibration even with using sparse $3 \mathrm{D}$ point clouds as shown in figures $(5,8)$, it is expected to achieve higher system calibration accuracy when using higher sensors configuration which can provide more dense 3D point clouds either by including more cameras or by using a more dense LIDAR scanner.

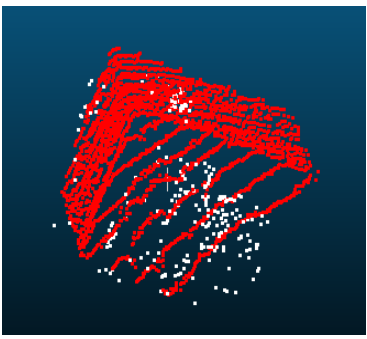

Figure 9.a

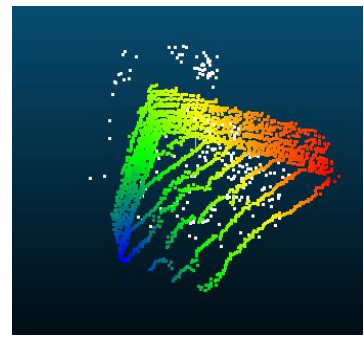

Figure 9.b (a). The point clouds constructed from the images and the registered LIDAR using the estimated calibration parameters

(b). The point clouds constructed from the images and the LIDAR before calibration

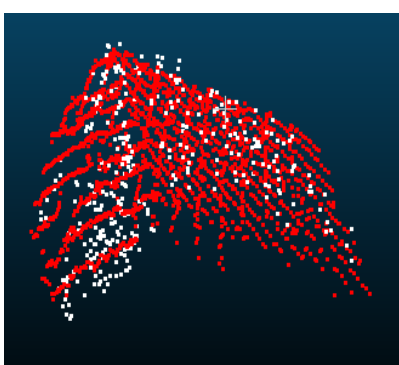

Figure 10.a

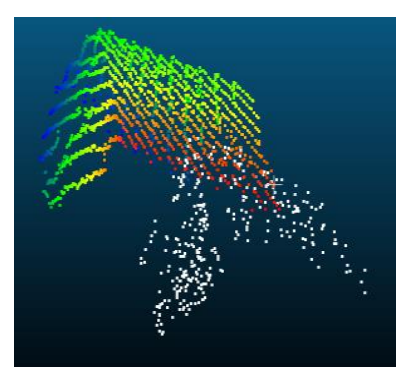

Figure 10.b (a). The point clouds constructed from the images and the registered LIDAR using the estimated calibration parameters

(b). The point clouds constructed from the images and the LIDAR before calibration

To test the effect of the distance between the target and the sensors, the target has been placed at two different distances in the third and fourth data sets in the second system scenario. In the third data set, the target was positioned 2.0 meters away from the laser scanner while the distance between them was 3.0 meters in the fourth data set. Following the same testing procedures, in the first case, the calibration parameters were estimated using the third data set and evaluated with the fourth data set. The testing was performed again in the second case using the fourth data set to estimate the calibration parameters and was evaluated using the third data set. The results of these two tests are shown in table (2). The results in table (2) show that the effect of the distance between the target and the sensors is very weak, as the mean and the standard deviation of the registration error is almost the same in the two cases.

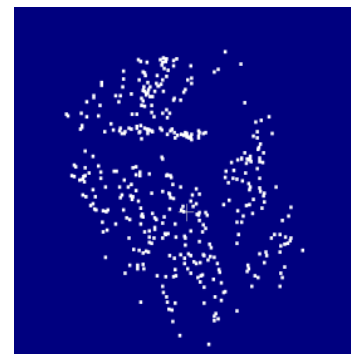

Figure 11.a

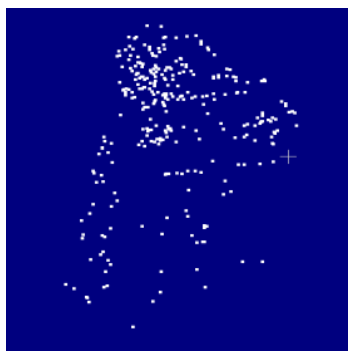

Figure 11.b (a) 3D point cloud from the first data set (b) $3 \mathrm{D}$ point cloud from the second data set

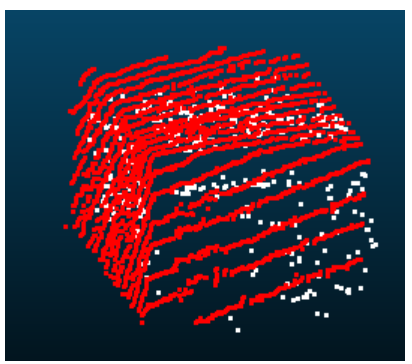

Figure 12.a

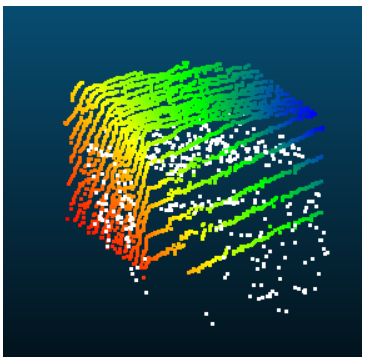

Figure 12.b (a). The point clouds constructed from the images and the registered LIDAR using the estimated calibration parameters

(b). The point clouds constructed from the images and the LIDAR before calibration

In the first scenario, it was noticed that the constructed 3D point cloud of the images for the target from the second data set was providing less points compared to the first data set as shown in figure (11). So, to test the ability of the proposed technique to deal with the shortage of points, the second data set was used to estimate the calibration parameters and the first data set was used to evaluate these parameters.

Figures (12.a, 12.b) show the results of the previous test which is tabulated in table (3). In table (3) the first case is when the calibration parameters were estimated from the first data set and the second data set was used to evaluate them, while the second case is showing the results of using the second data set to estimate the calibration parameters and evaluating them using the first data set.

\begin{tabular}{|c|c|c|c|c|}
\hline \multirow{2}{*}{ Case } & \multicolumn{2}{|c|}{ Case (1) } & \multicolumn{2}{c|}{ Case (2) } \\
\cline { 2 - 5 } & $\begin{array}{c}\text { Mean } \\
(\mathrm{m})\end{array}$ & Std (m) & $\begin{array}{c}\text { Mean } \\
(\mathrm{m})\end{array}$ & Std (m) \\
\hline $\begin{array}{c}\text { Before } \\
\text { calibration }\end{array}$ & 0.2960 & 0.1314 & 0.1555 & 0.0767 \\
\hline $\begin{array}{c}\text { After } \\
\text { calibration }\end{array}$ & 0.0644 & 0.0514 & 0.0585 & 0.0458 \\
\hline
\end{tabular}

Table 2. The mean and the standard deviation of the distance between the LIDAR and images-driven point clouds before the calibration and after the calibration for testing the distance effect 


\begin{tabular}{|c|c|c|c|c|}
\hline \multirow{2}{*}{ Case } & \multicolumn{2}{|c|}{ Case (1) } & \multicolumn{2}{c|}{ Case (2) } \\
\cline { 2 - 5 } & $\begin{array}{c}\text { Mean } \\
(\mathrm{m})\end{array}$ & Std (m) & $\begin{array}{c}\text { Mean } \\
(\mathrm{m})\end{array}$ & Std (m) \\
\hline $\begin{array}{c}\text { Before } \\
\text { calibration }\end{array}$ & 0.1067 & 0.061985 & 0.0975 & 0.0490 \\
\hline $\begin{array}{c}\text { After } \\
\text { calibration }\end{array}$ & 0.066 & 0.06484 & 0.0485 & 0.0234 \\
\hline
\end{tabular}

Table 3. The mean and the standard deviation of the distance between the LIDAR and images-driven point clouds before the calibration and after the calibration to test the cloud sparsity effect

The results of the previous test prove the ability of the proposed technique to keep calibration performance even with the low amount of the extracted points. This mainly can be explained by the collaborative fashion of the points that enables surface representation which in turn help the registration between the surfaces of the two point clouds.

\section{CONCLUSIONS}

A new calibration approach to estimate the geometric relationship between a LIDAR scanner and a multi-camera system has been proposed. The technique offers a convenient calibration procedure avoiding restrict arrangements, and using a simple target and minimal image acquisitions. The achieved results fit the accelerating needs for fast, and convenient system calibration methods.

It is important to note that the achieved results of the proposed technique can be improved by using more accurate sensors for constructing the 3D point clouds. The proposed system calibration technique depends on estimating the calibration parameters through the point clouds registration using the ICP, so more accurate $3 \mathrm{D}$ point clouds with less noise data will improve the registration step and consequently can help provide more accurate estimation for the calibration parameters.

\section{ACKNOWLEDGEMENTS}

This work was supported by Dr. Naser El-Sheimy research funds from NSERC and Canada Research Chairs programs.

\section{REFERENCES}

Bay, H., Ess, A., Tuytelaars, T., Van Gool, L., 2008. Speeded-up robust features (SURF). Computer vision and image understanding 110, pp. 346-359.

Besl, Paul J., and Neil D. McKay, 1992. Method for registration of 3-D shapes. Robotics-DL tentative. International Society for Optics and Photonics.

Douillard, B., Fox, D., Ramos, F., 2007. A spatio-temporal probabilistic model for multi-sensor object recognition, IEEE/RSJ International Conference on Intelligent Robots and Systems (IROS), pp. 2402-2408.

George Vosselman, H.-G.M., 2010. Airborne and Terrestrial Laser Scanning. CRC Press.

Heikkila, J., Silvén, O., 1997. A four-step camera calibration procedure with implicit image correction, IEEE Computer Society Conference on Computer Vision and Pattern Recognition, pp. 1106-1112.
Kwak, K., Huber, D.F., Badino, H., Kanade, T., 2011. Extrinsic calibration of a single line scanning lidar and a camera, IEEE/RSJ International Conference on Intelligent Robots and Systems (IROS), pp. 3283-3289.

Kwak, K., Huber, D.F., Chae, J., Kanade, T., 2010. Boundary detection based on supervised learning, IEEE International Conference on Robotics and Automation (ICRA), pp. 3939-3945.

Lin, Y., Hyyppä, J., Jaakkola, A., 2011. Mini-UAV-borne LIDAR for fine-scale mapping. Geoscience and Remote Sensing Letters, IEEE 8, pp. 426-430.

Rodriguez, F., Sergio, A., Frémont, V., Bonnifait, P., 2008. Extrinsic calibration between a multi-layer LIDAR and a camera, IEEE International Conference on Multisensor Fusion and Integration for Intelligent Systems (MFI), pp. 214-219.

Vallet, J., Panissod, F., Strecha, C., Tracol, M., 2011. Photogrammetric performance of an ultra light weight swinglet UAV, $U A V-g$.

Zhang, Q., Pless, R., 2004. Extrinsic calibration of a camera and laser range finder (improves camera calibration), IEEE/RSJ International Conference on Intelligent Robots and Systems,.(IROS), pp. 2301-2306.

Zhang, Z., 2000. A flexible new technique for camera calibration., IEEE Transactions on Pattern Analysis and Machine Intelligence, pp. 1330-1334. 